\title{
A Novel Liquid Ionic Antenna for Bio-signal Monitoring Applications
}

\author{
*Anya Traille ${ }^{1,2}$, Li Yang ${ }^{2}$ and Manos M. Tentzeris ${ }^{2}$ \\ Email: anya.traille@gtri.gatech.edu \\ ${ }^{1}$ Sensors and Electromagnetic Applications Laboratory \\ Georgia Tech Research Institute, Atlanta, GA 30332, USA \\ ${ }^{2}$ GEDC, School of ECE, Georgia Institute of Technology, Atlanta, GA 30332, USA
}

Abstract - The performance of most commonly used metal antennas close to the human body is one of the limiting factors of the performance of biosensors and wireless body area networks (WBAN). Due to the high dielectric and conductivity contrast with respect to most parts of the human body (blood, skin, ...), most of the wireless sensors operating in RF and microwave frequencies is limited to $1-2 \mathrm{~cm}$ when attached to the body. In this paper, we introduce the very novel idea of liquid antennas, that is based on the conductivity of aqueous solutions of salt. This approach allows for the improvement of the range by a factor of 5-10 in a very easy-to-realize way, just modifying the salinity of the aqueous solution of the antenna. A similar methodology can be extended to the development of liquid RF electronics for implantable devices and wearable real-time biosignal monitoring, since it can potentially lead to very flexible antenna and electronic configurations.

\section{INTRODUCTION}

The explosive growth of the biosensors and health-related wearable monitoring devices has accentuated the need for miniaturized, high-efficiency conformal materials that can operate over a wide range of frequencies, while they can be integrated in wearable and lightweight configurations. Metallic antennas do not operate sufficiently when planted extremely close to the human body due to the dielectric discontinuity against human tissue (Metal: $\varepsilon_{\mathrm{r}}=1$, Blood: $\varepsilon_{\mathrm{r}}=58$, Skin: $\varepsilon_{\mathrm{r}}=37$ ) that causes the disruption of their near field. The problem of matching 'human tissue' to 'air' is commonly encountered in ultrasound techniques, which led to the research and development of tissue mimicking dielectric phantom models. In addition to matching, metallic antennas are heavy, vulnerable to corrosion, toxic to the human body and bending them introduces unwanted resonances. Liquid Antennas on the other hand, enclosed in glass would possess the biocompatible properties that would be useful for health monitoring devices, especially when they are implanted into human tissue. Plus, liquid (e.g.aquatic) solutions can be enclosed in flexible plastic, and bent in various configurations without introducing holes or air gaps, thus allowing them to operate sufficiently while worn as clothes. Liquid antennas would also be smaller as well as lighter allowing them to be easily integrated into everyday mobile human activities.

Material Characterization: Dielectric Liquids: The first step for the realization of practical liquid antennas is the accurate determination of how, fluid composition (ion species), electrolyte concentration, electrode polarization, geometry (e.g. toroid curvature), transport resistance, frequency and other parameters will affect the current distribution, radiation pattern, and efficiency of the liquid antenna. It is essential to characterize the electric properties of various electrolytes, however complicated is the process both theoretically and experimentally. As of now, approximations of electrical 
properties can be derived using a combination of Force Field equations, MD (Molecular Dynamics) Simulators, Debye or Cole-Cole theories of molecular relaxation as well as some experimental data to create empirical models that characterize the trend. Measurement uncertainties are discussed later in the paper.

Adding salt reduces the static permittivity (from 80 down to $30-40$ sometimes) of aqueous electrolyte solutions due to several effects. The easiest to conceptualize is solvent dipole density dilution. Solvent (e.g. water) molecules bond to the ions cancelling out their dipole moment. Another effect is the hydrodynamic rotation of the water molecules that results from the ion migration under the influence of the electric field.
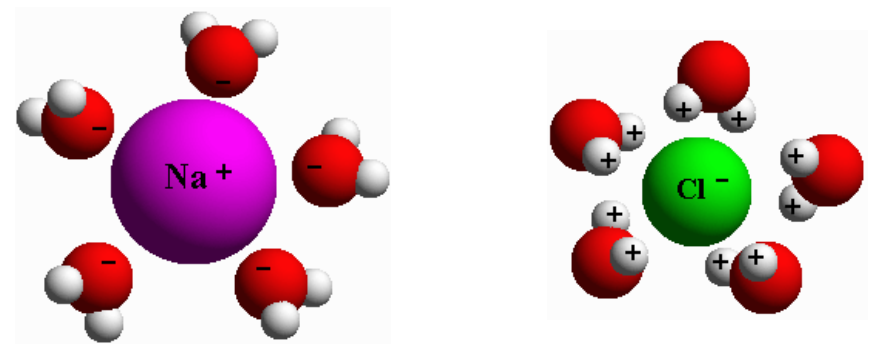

Figure 1. $\mathrm{Na}^{+}$and $\mathrm{Cl}^{-}$ions in water.

Principle of Operation: In electrolyte solutions, current is created by ions which migrate under the influence of an electrical field. In the case of an electrolytic liquid-filled loop antenna, the voltage gradient is due to the Lorentz force, that can be generated by two electrodes connected on opposite sides of the loop, similar to that of a toroid shaped battery. The antenna must be designed and tuned so that the charge-discharge-charge cycle occurs at a specific resonant frequency, that determines the antenna frequency.

\section{RESULTS}

As a proof of concept, free-space, as well as in Vitro (in the proximity of a "SEP" human head phantom) simulations benchmarking one liquid-loop antenna performance were performed using FEKO. The antenna geometry consists of a dielectric toroid operating at $915 \mathrm{MHz}$ with an aquatic solution $5 \mathrm{~mol} / \mathrm{L} \mathrm{NaCl}$, that is connected with an edge port between two hollow metal plates.

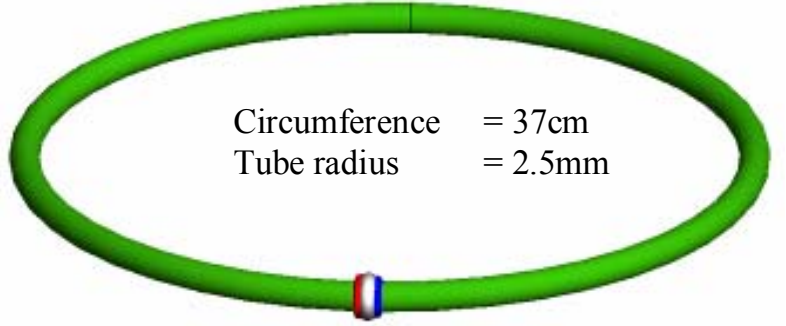

(a)

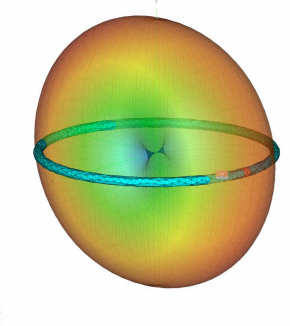

(b)

Figure 2. a) Ionic Loop Antenna b) E-Pattern (Free space). 


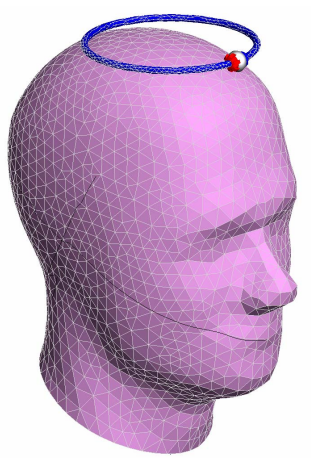

Figure 3. a) Human Head in FEKO (Skin $\left.\varepsilon_{\mathrm{r}}=41, \tan \delta=0.414 @ 915 \mathrm{MHz}\right)$.
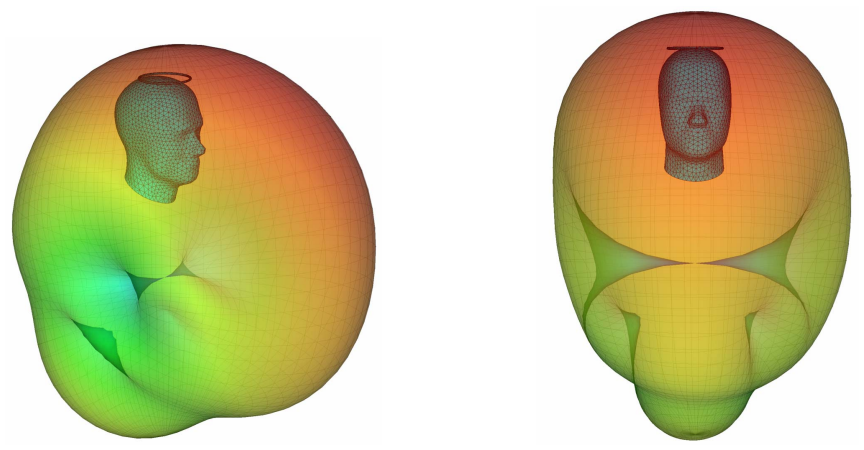

Figure 4. Liquid Antenna on "SEP” Human Phantom $\left(\mathrm{NaCl} \varepsilon_{\mathrm{r}}=40, \tan \delta=0.175 @ 915 \mathrm{MHz}\right)$.
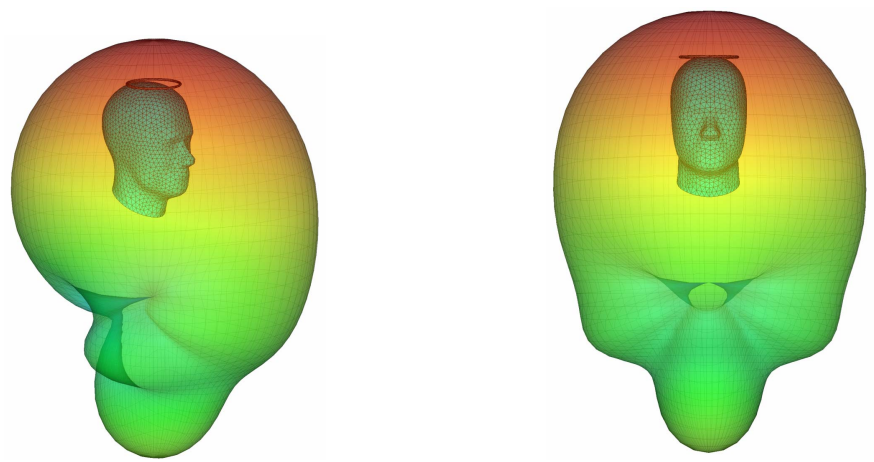

Figure 5. Metallic antenna on "SEP" Human Phantom $\left(\mathrm{PEC} \varepsilon_{\mathrm{r}}=1\right)$.

The radiation pattern (Fig.4) was compared with that of a metallic loop antenna with the same dimensions (Fig.5). It is clear that the liquid antenna pattern is much more concentrated in the area of the phantom eliminating stray radiation and enhancing "highfocus" biodiagnostic applications. In addition, the material "near-field" matching capability of the liquid antenna can be readjusted "on the fly" for positioning close to different tissues by modifying the molarity of salt, thus changing the dielectric constant, something impossible for the conventional metal antenna.

Feeding \& Measurement: Electrode-Liquid Interface: The feeding will be a non-faradic electrode process, using an IPE (Ideal Polarized Electrode), where no charge transfer will occur between metal and liquid. Since the ions cannot penetrate the metallic-liquid interface, they will accumulate at the electrodes forming an ionic layer commonly 
referred to as the Electrical Double Layer (EDL). The EDL consists of absorbed and solvated ions creating a double layer capacitance between the electrolyte solution (charged ions) and metal, as well as between charged particles and electrolyte solutions (colloidal dispersions). EDL capacitance affects the electrode process by creating voltage gradients that alter the current measured by external circuits. The DL thickness changes with concentration as well as other parameters beyond the scope of this paper. Its effect, it is less pronounced at high frequencies however cannot be ignored when characterizing dielectric liquids for their electrical properties. Detailed experimental results will be presented at the conference for a variety of solutions, operation frequencies and tissues.

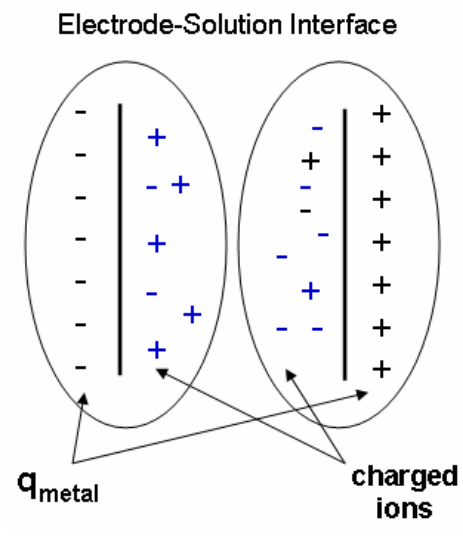

Figure 6. Electrode-Solution interfaces

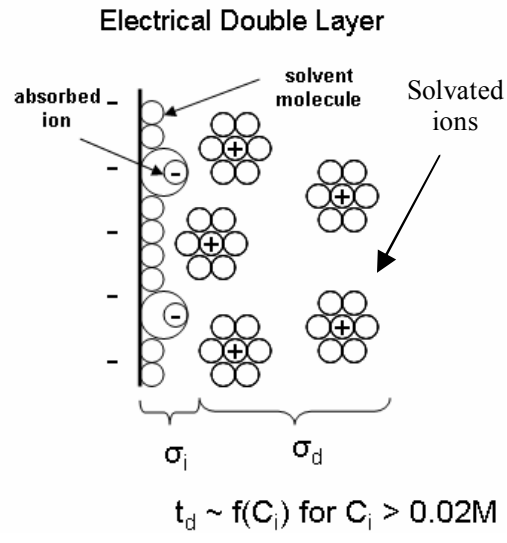

Figure 7. Electrical Double Layer

\section{CONCLUSIONS}

We have demonstrated for the first time in RF frequencies the capabilities of the novel concept of liquid antennas for wearable bio-monitoring applications. Although conventional metal antennas suffer from low range and oxidation problems when in proximity to the human body, the proposed topology features an improved range by an order of magnitude. In addition, it is very conformal allowing for wearable WBAN implementation, as well as it can be very easily reconfigured by adjusting the salinity of the salt solutions. A benchmarking prototype has verified our preliminary simulation conclusions. The proposed work could set the foundation for the implementation of truly wireless biosensor networks with small size, lightweight conformal nodes for wireless health monitoring in pharmaceuticals, hospital, ambulance and home-based patient care.

\section{REFERENCES}

[1] B. Allen, L Faulkner, "Electrochemical Methods: Fundamentals and Applications," Department of Chemistry and Biochemistry University of Texas at Austin, John Wiley \& Sons, Inc, 2001.

[2] S. Gabreil et al., "The dielectric properties of biological tissues: III. Parametric models for the dielectric spectrum of tissues," Physc. Med. Biol, 1996, pp 2271-2293.

[3] K. Nortemann, J. Hilland, U Kaatze., "Dielectric Properties of Aqueous $\mathrm{NaCl}$ Solutions at Microwave Frequencies," J. Phys. Chem. A 1997, 101, 6864-6869. 